\title{
L'ORALITÉ DANS LA COMMUNICATION ASYNCHRONE VIA INTERNET SUR LES EXEMPLES DU DISCOURS DU CYBERESPACE FRANÇAIS ET ITALIEN
}

\begin{abstract}
Pirogowska Ewa, Loralité dans la communication asynchrone via Internet sur les exemples du discours du cyberespace français et italien [Orality in the asynchronous communication via Internet on the examples of discourse in the French and Italian cyberspace]. Studia Romanica Posnaniensia, Adam Mickiewicz University Press, Poznań, vol. XXXIII : 2006, pp. 181-197. ISBN 83232-1643-6, ISSN 0137-2475.
\end{abstract}

The aim of the article (based on the ffragments of the doctoral dissertation) was the analysis of the spoken language functioning in the Italian and French cyberspace as a communication system secondary to the oral skills (orality) of the first human groups. The thesis is the following: in the clectronic communication the two language uses, the written and the spoken, cannot be treated separately, as a dichotomy, as they coexist and are connected by one common code, "work" language, Computer Mediated Communication. The communication presented is devoted to the conversational analysis of discourse in the asynchronous communication, the dialogue exchange on forums and discussion groups. The notion-keys are as follows: asynchrony of dialogue microspace, inter-subjective space, sequential implicativness, conversational strategy.

Les participants de l'échange conversationnel dans le cyberespace peuvent difficilement utiliser des éléments de la situation énonciative (comme par exemple la réaction de l'interlocuteur au message émis), autrement que dans le cas de l'interaction face à face. Le sujet parlant se sent souvent anonyme et dispensé du souci de l'authenticité et c'est dans ce cadre que le média observé détermine le type d'interaction qui se produit. Dans le présent article, nous tenons à présenter quelques observations sur le fonctionnement de l'échange asynchrone des messages électroniques qui se produit dans les espaces dialogiques de forums de discussion dont le fonctionnement ressemble en plusieurs points à l'échange de correspondance via e-mail. Le participant d'une discussion reste plus ou moins anonyme, s'il ne se présente pas directement, il ne dévoile son identité qu'à travers le sous-entendu de l'énonciation.

Dans les travaux de Fabienne Cusin-Berche, nous rencontrons le terme de discours reporté (Cusin-Berche, 1999) créé pour expliquer et décrire la possibilité, 
offerte par le logiciel de type Outlook Express, d'introduire le texte entier ou une partie du message reçu antérieurement dans le corps de la réponse. Cette dénomination " permet de désigner un objet textuel construit à partir d'une multiplicité énonciative dialogale qui ressemble à une situation conversationnelle et qui s'en distingue par sa matérialité graphique, laquelle implique une segmentation, voire une sédimentation discursive » (Cusin-Berche, op. cit. : p. 51, cf. Mondada, 1999). Nous avons donc ici une comparaison du message électronique composé d'un énoncé initial et de réponses à l'échange des communiqués oraux. Le point commun de ces deux types de communication est que le sens global ne se construit que si l'on prend en considération la totalité des énoncés particuliers qui se succèdent; le sens se construit toujours en contexte. Les énoncés de l'exemple présenté ci-dessous :

\section{La réalisation de 1 'ouvrage est en cours qui publie ces carnets}

seraient donc difficilement compréhensibles sans le procédé de reporter-réinsérer des éléments des messages antérieurs. Les indices linguistiques qui permettent de localiser le contexte, sont pour Cusin-Berche (op. cit. : p. 52) les désignateurs. Il s'agit des articles définis, adjectifs possessifs et démonstratifs ; les marqueurs épistémiques ("Chères Mesdames ») et chronologiques. Cusin-Berche parle aussi du principe de coopération', une logique de la communication : un échange de mails peut se produire entre les deux sujets en relation communicative justifiée par la situation.

Le message électronique particulier devient ainsi un micro-espace dialogal asynchrone; les énoncés-apports dialogiques sont ${ }^{2}$ ajoutés successivement. La multiplicité dialogale du courrier électronique, créée par les énoncés groupés en séquences, nous conduit à penser aux tours de parole, terme propre à l'analyse conversationnelle. Les séquences dialogiques, à l'image des tours de parole, rapprochent l'e-mail d'un fragment de la conversation à vive voix et assurent une continuité à un échange de courrier électronique (cf. Hert, 1997 :27). Orlikowsky et Yates (1994) ont même défini un genre des messages électroniques qu'ils ont appelé dialogique ; Cusin-Berche parle du messiel (Cusin-Berche, 1999 : 54). Pourtant, il faut l'admettre, c'est la perspective de la matérialité graphique où l'échange de lettres électroniques s'avère être plus proche de la correspondance traditionnelle, comprise en tant qu'un échange spécifique des messages, réparti dans le temps, que de la conversation à vive voix. Lors de cette dernière, soit par téléphone, soit en direct (l'espace dialogique de la t'chatche), le locuteur est renseigné soudainement quant aux dispositions réceptives de son allocutaire. Ceci est réalisé grâce aux

\footnotetext{
I Terme grâce au P.H. Grice (1979), Logique et conversation, Communications, $n^{0} 30$, Seuil.

${ }^{2}$ Ou plutôt : «peuvent être », parce qu'en général les internautes ne renvoient pas des messages initiaux, l'usage veut que l'on le fasse seulement dans le cas important, justifić par les circonstances, p.cx. l'observation fidèle du fil de la discussion.
} 
indices tels que le ton ou la suspension de la voix, les gestes et grimaces éventuels, etc. Dans le registre écrit interactif ce seront leurs substituts graphiques, comme smileys, sylabogrammes, interjections et emploi des majuscules (pour imiter l'émotion). La conversation («le flux discursif») peut donc être modifiée, ménagée à chaque moment, ce qui n'est pas le cas d'un échange de mails ou de lettres. L'embrayage du discours de l'e-mail ressemble à celui d'un échange oral, mais son fonctionnement référentiel est contextuel et non situationnel, l'embrayage n'a pas valeur de déixis - il ne peut pas sélectionner et montrer, par une mimique (geste du doigt, direction du regard), un élément de réalité dans le champ visuel des sujets communiquants (cf. Cusin-Berche, 1999 : 53). Il convient de dire que l'e-mail, au plan du fonctionnement référentiel, appartient au genre épistolaire. Cependant, comme le disent Orlikowsky et Yates, il existe une interdépendance entre les messages - cette fonctionnalité, difficile à produire à travers un support de papier, permet de faire des connexions entre messages.

Eu égard à la matérialité graphique de la correspondance via Internet, ce type de communication subit des contraintes spatiales et temporaires et devient, ou plutôt peut devenir (si l'on a affaire à un véritable échange de la correspondance, pas à une diffusion occasionnelle p.ex. du mailing), une succession tout à fait séquentielle des informations - où les séquences sont différées dans le temps. Sans une réponse sous forme de commentaire, l'e-mail reste un communiqué isolé et ne change pas en forme dialogale. D'autre part, si nous admettons que la lettre électronique devient, à la condition d'employer un discours reporté, un échange dialogal, il y a pourtant de l'artificiel dans la continuité intérieure du message e-mail. Comme il n'est pas possible pour l'émetteur de ménager à chaque moment le flux discursif, la réalisation de la réplique reste toujours éventuel, probable, dépendant de la situation de réception dans laquelle se trouve le récepteur. Le message peut être suivi d'une réplique, mais il se peut qu'il reste sans réponse.

Revenons pour le moment à la construction du message électronique. Comme nous l'avons déjà remarqué, le courrier électronique (le système de forum de discussion inclus) offre une possibilité, introuvable ailleurs ${ }^{3}$, d'incorporer la réponse, ou ses citations particulières, dans l'intégralité de l'émail - le message électronique peut devenir donc une sorte du discours reporté. Du côté technique, le phénomène consiste en recopier et coller un texte au sein de la réponse, ce qui s'effectue le plus souvent (de manière automatique) par la mise en relief de la partie insérée par le symbole $>$, le texte introduit étant placé généralement au-dessous de la réponse. A chaque reprise, le message reporté reçoit la marque $>$, donc il est facile à en déduire combien de fois ce processus, simulant des tours de parole, a été répété. Il se forme ainsi une sorte de polyphonie. Mais cette référence peut également se faire de manière indirecte, lorsque des participants sont cités dans un message par leur nom

${ }^{3}$ La construction des messages de la boîte postale des téléphones portables prévoit quand même l'inclusion du texte original ; le procédé semble être rare chez les utilisateurs vu l'espace limité du texte. 
et à travers un résumé de leurs positions. De la pratique de la citation, on passe ici à une pratique de synthèse. De manière générale, ces pratiques de citation de messages ont des influences sur la compréhension collective du débat.

L'expression spontanée des mails fait naître une affectivité aiguë et des scories (cf. Cusin-Berche, op. cit.), éléments linguistiquement parasites. Cela résulte de la manière de rédiger « au fil de la plume », " avec des abréviations personnelles parfois obscures à la lecture » (Mourlhon-Dallies et Colin, 1995 : 163). En ce point le flux discursif du message écrit provenant de la communication asynchrone s'apparente à l'énonciation orale, pleine d'expressions affectives qui n'apportent pas de sens à la totalité d'expression, mais témoignent de la forte tension émotionnelle, comme dans la contribution A.V.16 de notre corpus (Pirogowska, 2005) :

16. $(\ldots)$

- Baldoni ?

..Le simone, le simone... e perche' per Baldoni non e' stato fatto nulia?

$E^{\prime}$ una vergogna.

Le message sous cette forme ressemble à un énoncé oral, écrit ensuite en hâte sur une feuille de papier. Le caractère émotionnel prévaut sur l'informativité.

\section{ORGANISATION SÉQUENTIELLE DU DIALOGUE ASYNCHRONE}

L'opinion répandue parmi les analystes du langage d'Internet est que l'échange des messages où que ce soit (courrier électronique, forum de discussion, causettes) exige d'autres compétences que les échanges communicatifs dits traditionnels, qu'il " ne sollicite ni ne nourrit la même compétence que l'écriture d'une lettre ou d'un journal interne ou que la pratique orale du débat en classe " (Mondada, $1999: 3$ ). Cependant les études montrent qu'il y a des pratiques qui se prêtent parfaitement à la communication par le biais de nouveaux médias comme Internet ou le téléphone mobile. Le courriel est une manifestation caractéristique du registre interactif écrit, mais à la fois spécifique, puisqu'il est asynchrone, il tente de se rapprocher du temps réel (cf. Anis, 1999 : 57). L'émission du message, bien qu'immédiate, doit être suivie de sa réception dont le moment n'est pas tout à fait à prévoir. Nous voudrions réfléchir et essayer de répondre à la question, en profitant largement des études menées par Lorenza Mondada (op. cit. : 3-25) quelle organisation séquentielle et interactionnelle caractérise les messages asynchrones comme les courriels et les messages échangés lors de discussions dans Internet, qui constituent actuellement les formes les plus pratiquées de cybercommunication. Nous avons trouvé pertinent de nous occuper de la séquentialité des messages asynchrones parce que cette organisation discursive approche un échange des messages via Internet d'une conversation quotidienne à vive voix. Notre avis est que c'est dans l'organisation séquentielle de 
l'échange asynchrone de messages que l'oralité du langage d'Intemet se manifeste pleinement.

Nous voudrions préciset en ce lieu que pour les buts d'analyse conversationnelle nous ne ferons pas la différence concernant la nature technologique de la diffusion des messages asynchrones. L'idée de l'échange communicatif, quel que soit le moyen de présentation - la boîte aux lettres de type Outlook Express ou bien le forum de discussion d'un site Internet, est en principe la même. Les différences existent au niveau de l'interlocuteur, de l'énonciataire. S'il est individuel ${ }^{4}, l^{\prime}$ 'interaction se joue au niveau interpersonnel ; si, en revanche, il constitue un groupe multi-participants, l'interaction devient publique, elle devient donc un vrai forum. Nous pouvons cependant mentionner qu'il arrive qu'une discussion entre les deux participants se déclenche suite à un propos incitant à la réaction; les autres users l'observent sans trop réagir. Tel est le cas, par exemple, de la discussion en sein du forum entre vincenza \& francesco et paola baldi, partie A.V de notre corpus (Pirogowska, 2005). Les internautes échangent des opinions sur la libération de deux femmes kidnappées en Iraq; le conflit se déclanche avec l'expression beduini arrappati et le fil conducteur de l'échange entre les users du forum se concentre sur la compétence langagière des participants. Les indices intratextuels dénoncent, entre autres, la volonté de s'exprimer librement par l'internaute Paola, qui accuse Vincenza et Francesco d'être des catholiques entêtés, et l'emploi incorrect du verbe italien arrappare. Le dernier problème est important pour les intervenants, parce que la compréhension inexacte du terme beduini arrappati pour désigner les islamistes iraquiens semble être à l'origine de la querelle. Le cas présenté nous semble être assez typique; dans les discussions, surtout asynchrones (car elles permettent d'étaler les réflexions sans limite de temps et d'espace), le petit malentendu peut provoquer les essais de précision du terme qui vont jusqu'au raisonnement sur l'étymologie des mots, leur champ sémantique et l'acquisition des synonymes. Nous avons déjà remarqué que dans internet on n'a pas de possibilité d'observer les réactions de l'interlocuteur comme dans le dialogue in praesentia. Comme la situation énonciative de nos exemples ne le permet aucunement - les indicateurs non verbaux sont ici pratiquement absents - les sujets interagissant ne sont pas toujours capables de distinguer les nuances sémantiques. Il arrive donc qu'un seul mot, une faute d'orthographe (témoignant par exemple de l'ignorance éducative) ou une intervention originale entraîne une suite énonciative agressive. L'échange d'opinion de forum n'est sûrement pas moins violent qu'une discussion réelle; parfois l'achamement des participants s'avère plus fort vu qu'ils restent anonymes, donc plus courageux et libres de s'exprimer.

\footnotetext{
${ }^{4}$ Même si le message est adressé à un groupe de personnes, la réception est individuelle, c'est-àdire chacun le reçoit à son compte.
} 


\section{ESPACE INTERSUBJECTIF ET IMPLICATIVITÉ SÉQUENTIELLE}

La règle est que l'internaute reprend le message de l'autre pour y répondre : il fait ses commentaires, le complète etc. Ainsi on voit apparaitre un certain effet de loupe - le fragment commenté du message est mis en relief en tant qu'appui à la séquence suivante. «Pour cette façon de traiter le discours de l'autre, l'énonciateur introduit une interactivité dans son message et il la met en scène : il rend aussi manifestes ses activités de production et d'interprétation ; il construit un espace d'intersubjectivité qui pourra être modifié par les locuteurs successifs » (Mondada, 1999 : 5). Cet espace d'intersubjectivité se voit remanié dans les chaînes de contributions de forums de discussion, où le point de vue de l'internaute lanceur du thème est commenté par les locuteurs successifs. Une analyse du phénomène est proposée dans notre récente étude, L'oralité secondaire dans le discours du cyberespace français et italien, p. 107-123.

L'interactivité, trait caractéristique des échanges à vive voix, est la plus importante propriété de tous les discours d'Internet, mais conformément à un type particulier du discours, la dimension interactive peut être différente. Nous voudrions présenter en ce lieu l'état des recherches sur l'interaction dans les échanges asynchrones sur Internet - les mails et, puisque les mécanismes sont les mêmes, aussi les contributions des forums de discussion.

Ce qui caractérise sûrement les débats en ligne, qui se sont déroulées dans des forums de discussion, c'est que les participants tâchent à construire un sens d'un vrai échange d'idées et, à travers la langue écrite, ils créent un sens du collectif. Le problème a été largement étudié par Philippe Hert (1997). Il est intéressant de se pencher sur l'énorme difficulté dans la construction d'un sens commun à travers les interactions électroniques, en ce lieu celles qui sont appelées asynchrones. Elles sont évidemment plus faciles à observer (en tant que facilement enregistrables) par rapport aux dialogues de t'chatche, mais à la fois toujours plus longues et d'un contenu plus complexe.

Nous avons voulu montrer de façon détaillée l'organisation séquentielle de l'enchaînement des messages envoyés selon le schéma nommé dans la littérature paires adjacentes, par exemple question/réponse, jugement/commentaire, salutation/salutation, invitation/acceptation ou refus. Quoique la notion de paires adjacentes soit liée à l'analyse conversationnelle des échanges synchrones, face à face entre les deux locuteurs, nous nous permettons de la prêter pour pouvoir comprendre le mécanisme discursif de l'échange des mails, opération asynchrone. La séquentialité s'agence suivant le principe des tours de parole, une autre notion empruntée à l'analyse conversationnelle. Pour faire avancer nos observations, nous avons choisi ces messages qui ont formé un véritable échange des communiqués successifs et créé une suite logique et cohérente. Puisque les messages échangés sur des listes de discussion ou dans des forums de discussion constituent un exemple spécifique de 
la forme plurilogale, il est intéressant de révéler les traits propres de la construction du sens global.

Il y a quelques éléments qui doivent être rappelés. Dans un message asynchrone nous avons souvent affaire au discours reporté, comme cela vient d'être dit. Il est construit à la base du procédé utilisé fréquemment qui consiste en la reprise d'un élément (mot, groupe de mots, phrase entière ou coupée - sous forme de citation) du message précédent pour en faire l'appui à la construction de la réponse. Le procédé est caractéristique pour le courrier électronique, dans la façon que l'on répète et incorpore d'assez longs fragments du message initial ; les messages postés dans les forums contiennent de simples citations de mots employés ou des messages antérieurs intégraux, incorporé par le système de publication en ligne d'un site donné. Si nous nous souvenons de la définition de Cusin-Berche (1999: 51), nous pouvons dire que grâce à la construction du discours reporté l'échange des mails ressemble à une discussion réelle ${ }^{5}$. En même temps, le procédé de répétition des fragments de texte qui fait d'un message une compilation des contributions antérieures (la matérialité graphique de Cusin-Berche) ne permet pas d'oublier la spécificité de ce genre de communication. Pour être compréhensible, un e-mail doit être souvent segmenté, sédimenté même.

Dans notre corpus (Pirogowska, 2005 : surtout A.II) la volonté d'être bien compris et bien situé dans la discussion est visible. Certains internautes n'abandonnent pas les propos antérieurs, mais ils ajoutent simplement leurs réponses à tout ce qui avait été déjà dit. Ceci conduit à la production des chaînes discursives, où les fragments - messages individuels - sont différenciés à l'aide des intervalles paralinguistiques (régis par le système) comme par exemple:

a) mary anne <maryanne54@y...> wrote :

b) re :

c)

Ainsỉ le lecteur bien orienté qui suit l'enchaînement des fils discursifs dans un forum thématique, sursaute au dessus des parties textuelles incluses en tant que le discours reporté.

3620De: Alain H. <alain033@y...>

Date: Lundi, 4. Octobre 2004 1.4: 49

Objet: Re: Réf.: Re: [devenons amis] Bonjour

bonjour maryanne merci jai passer un bon dimanche jespere que toi aussi je continue un voilier que jai commencer ya un mois je les presque finit ses pas que je culpabilise mais quelque part si enfin je suis comme sa oui je me deplace je sort car jai un musee il faut bien s ent occuper

${ }^{5}$ Nous comprenons l'adjectif qualificatif « réel " toujours comme l'antonyme de "virtuel " et non dans l'opposition à « fictif », « irréel ». 
mary anne <maryanne54@y...> wrote: bonjour, Alain! j'espère que tu as passé un bon

dimanche, qu'est ce que tu as fait ? la vie nous réserve

des joies mais aussi des peines, il ne faut pas te

culpabiliser de ce qui est arrivé à ta femme, c'est

ton trvail qui t'imposait a'être loin et elle était

certainement fragile.est ce que tu peux te déplacer

depuis ton accident ? a plus, maryanne

re bonjour mary moi aussi jai trois petit enfants

es deux grande fille vive maritalement et habite pres

de chez moiet ma derniere et avec moi car jai divorcer

car mon ex femme buvais quand jetais pas la oh je lui

en veux pas ses de ma faute si javais etais a la

maison plus souvent au lieux de revenir qune fois par

moi je pense que sa ne serais pas arriver mais comme

je travailler a 1 etranger ses pas facille de revenir

de aussi loint au plaisir de te lire alain $h$

mary anne <maryanne549y... > wrote :

Alain, merci pour ton mail; moi j'ai 4

enfants : madeleine 29 ans, alexandre 25 ans, emilie 22

ans et noemie 14 ans; bien sûr, les grands sont mariés

et je suis "grand mère" 5 fois ! c'est incroyable, 500

films !! c'est une belle collection; j'aime aussi

beaucoup le cinéma, sinon j'aime la lecture, la nature

et Ies animaux ; tu dois être trés patient pour faire

des maquettes.tes filles sont avec leur maman ? tu les

vois souvent ? passe un bon dimanche et à

bientôt, maryanne

La succession temporelle n'est ici que la relation naturelle, fondement du phénomène de séquentialité, lequel est construit autour de dépendances résultant du fait que le deuxième message incorpore le premier. Et ce faisant, exhibe les contraintes que le premier exerce sur lui tout en les configurant en retour par les choix de segmentation effectués (Mondada, op. cit. : p. 6) :

succession temporelle :

message $\mathrm{A}$ - message $\mathrm{B}$

organisation séquentielle du message $B$ :

[citation du A / reprise B] [citation du A / reprise B] [citation du A / reprise B] 
La séquentialité déploie ici son rôle structurant - les relations qui s'établissent dans la temporalité de l'altemance de la parole d'un locuteur à un autre sont en effet constructives pour son organisation. L'interlocuteur, dans son intervention, complète le message initial d'un nouveau contenu sémantique, mais procède aussi à la création des attentes et contraintes normatives. Ces conditions, qui gèrent tout échange communicatif, ont été appelées par Schegloff et Sacks l'implicativité séquentielle, sequential implicativeness (Schegloff, Sacks, 1973 : 289-327, cités aussi par Mondada). L'implicativité séquentielle organise le fonctionnement des paires adjacentes que nous avons mentionnées - les deux parties sont reconnaissables par les sujets communiquants : «A basic rule of adjacency pair operation is: given the recognizable production of a first part, on its first possible completion its speaker (pour nos besoins speaker sera l'émetteur du message ou co-scripteur, coscritto) should stop and a next speaker should start and produce a second pair past from the pair type of which the first is recognizably a member " (Schegloff, Sacks, op. cit. : p. 296). Chaque tour d'un échange des énoncés produit un effet rétrospectif et prospectif, sur quoi est fondée l'organisation séquentielle. L'interlocuteur ou les interlocuteurs (forum de discussion) qui créent la suite du message, complètent l'enchaînement séquentiel de l'échange communicatif. Le récepteur du message repère dans son texte les unités analogues aux premières parties de la paire adjacente qu'il reproduit dans la réponse sous forme de citations (pour souligner que c'est le discours de l'autre) et construit la seconde partie de la paire adjacente, la réaction à la première. L'incorporation des éléments du message initial rend compte des opérations que l'interlocuteur effectue sur le texte initial. Nous voyons apparaître les fragments du texte auxquels l'interlocuteur réagit parce qu'il les trouve importants, intéressants, pertinents. L'implicativité séquentielle est en rapport incessant avec le processus d'interprétation en contexte. Le locuteur second, récepteur du message, doit comprendre le communiqué conformément aux intentions de l'émetteur présentées lors du premier tour. C'est le deuxième tour qui en est la preuve. En même temps le locuteur second doit agir $=$ répondre conséquemment aux contraintes projetées par l'énoncé du premier tour. Le troisième tour rend cependant visible la réaction de l'émetteur du message original. En voyant les redondances de son texte lors du processus de la compréhension, il peut également observer ce que devient un même message chez deux locuteurs différents. Mondada souligne (op. cit. : p. 8) que la pratique de citation introduit dans le message les traces d'une interactivité à laquelle le message participe. La notion de séquentialité rapportée à l'alternance des tours de parole nous fait voir de près les différentes relations prospectives et rétrospectives qui naissent quand le second locuteur émet son message. Elles constituent les liens qui garantissent la continuité d'une série de messages.

De tout ce qui vient d'être énoncé surgit la question comment s'organise le processus public de la compréhension de la perspective du premier locuteur, toujours dans le spectre de l'analyse de l'enchaînement de messages électroniques. Il serait important de demander si les locuteurs-intemautes identifient facilement les 
points privilégiés dans le discours, s'ils participent activement dans la «co-construction » du message. Est-ce qu'il y a un contrôle de l'espace intersubjectif, de la compréhension générale de la discussion qui se déroule?

Prenons pour exemple la chaîne des messages A.II. de notre corpus ${ }^{6}$, un échange multi-participants asynchrone. Le matériel enregistré constitue le fragment d'un échange de contributions plus long, effectué entre les amis virtuels qui se partagent leurs opinions et racontent ce qu'ils font dans la vie. La contribution de $<$ Verron cath> A.II.14. déclenche les réactions des autres co-scripteurs. L'user <Verron cath>, femme de 58 ans, parle d'elle-même :

(...)je suis à la veille de prendre ma retraite(...)

(...) je suis présidente d'un lieu de vie pour enfants en difficultés (psychotiques, le plus souvent). C'est un rôle bénévole de gestionnaire (...)

Je suis aussi secrétaire d'une association qui parraine des enfants tibétains en exil (...)

et invite à continuer l'interaction -

(..) Qui s'intéresse à ces sujets ? Au fait, j'aimerai savoir, "a priori". ce que vous pensez du développement durable ?

Les co-scripteurs se voient inspirés de prendre la parole, parmi eux <Verron cath> A.II.15., <Utilisateur1> A.II.16 et <zahra ibn.> (A.II.17.). Les réponses des deux premières personnes sont motivées par des faits énoncés par <vernhes M.e>, dont la contribution est incorporée dans leurs répliques.

A.II.15: Je suis enseignante et donc sensible au sort des enfants. Je trouve ce que tu fais formidable (..).

A.II.16: la retraite ce n'estpas une si mauvaise chose

A.II.17: J'apprécie beaucoup tout ce que vous faites et je vous dis que vous n'allez pas du tout vous ennuyiez en étant en retraite

La contribution de <Verron cath> a produit un effet prospectif qui va organiser la séquence de réponses. Elle a impliqué la problématique à traiter par les locuteurs successifs : la retraite et l'aide aux enfants démunis de soins, ainsi que le soutien de contact. Le second tour de l'interaction s'organise selon les contraintes imposées par la retraitée. Malheureusement, nous ne connaissons ni la réponse de $<$ Verron cath $>$ ni la suite de l'interaction. La réponse de $<$ zahra ibn. $>$ est suivi de message de < abdelwhb t.h> A.II.19, pour qui le point de départ pour construire sa réaction énonciative est le travail de $\langle$ zahra $\rangle$. L'internaute $<$ abdelwhb $t . h>$ propose d'entreprendre la discussion sur le cote politico_toristique; cependant ce qui a suscité son intérêt c'est sans doute l'origine algérienne de $<$ zahra ibn.>

\footnotetext{
${ }^{6}$ Tous les exemples analysés proviennent de: Pirogowska (2005).
} 
Un fil antérieur de l'échange asynchrone A.II, conduit parallèlement, est plus développé. Il s'agit des contributions recueillies dans A.II.5 produit et émis par $<$ mary anne>, car elle incorpore dans sa lettre adressée aux amis virtuels tous les messages collés précédemment, lors de l'interaction à laquelle nous n'avons pas eu d'accès direct. La contribution A.II.5. est apparue telle dans sa forme intégrale dans le web, ressemblant à une grappe où les énoncés sont étagés sur un axe commun. Elle renferme en elle les strates discursives antérieures par rapport au message principal émis par <mary anne>. Les contributions postérieures sont les suivantes: A.II.6, 8, 9, 11, 13.

En ce lieu, mentionnons l'impact de Hert (1997: 6-7) sur notre étude. Le linguiste demande quelle est la construction sociale du sens du collectif propre à un groupe. Pour trouver la réponse, il a analysé l'énonciation dans l'espace électronique d'un groupe virtuel dans le forum de discussion qui avait également une existence sociale réelle (la communauté STS). Nous arrêtons nos recherches sur l'observation du comportement énonciatif des internautes dont le contact avec les autres interlocuteurs se limite aux rencontres régulières dans le net - tel semble être le cas de l'échange A.II. L'influence d'éventuelles réelles rencontres sur le comportement langagier des users de forum constitue la zone de notre ignorance. C'est une piste à dévoiler sans doute; dans le domaine des comportements langagiers au sein d'une communauté, nous nous sommes bornée à présenter en quelques mots la problématique de in-group language. Des questions importantes se posent sur le lien existant entre un collectif humain et les ressources qu'il peut mobiliser pour construire une compréhension collective. Hert constate que «c'est la parole qui circule au sein d'un collectif qui permet de construire ce sens commun. Que se passe-t-il lorsque cette parole fait défaut? »

Les internautes de A.II, qui apprennent à mieux se connaître, mènent en fait une conversation off line, alors dans les décalages de temps (pas trop longs, comme le montrent les données concernant la date et le temps de postage: A.II.5 - le 4 octobre, $14: 42$; A.II.6-le 4 octobre, 14:49; A.II.8-le 4 octobre, 17:56; A.II.9le 4 octobre, $18: 04$; A.II.11-le 5 octobre, 11:14; A.II.13-le 5 octobre, 11:40). Une autre discussion, à savoir A.V.1-27, apparaît comme un échange quasi-immédiat. Les horaires d'apparition des contributions en sont témoins; en fait les internautes participant à l'interaction ont posté les messsages en 4 heures (le fragment de la discussion enregistrée contient 27 contributions entre $18 \mathrm{~h} 41$ et $22 \mathrm{~h} 37$ du 30 septembre 2004). On peut admettre que le fil de la discussion a été suivi par tous les participants, même s'ils répondaient ponctuellement aux propos. Les liens qui s'établissent entre les contributions de ces deux forums permettent d'assurer la continuité de l'interaction. Parmi ces liens nous remarquerons le processus d'anaphore ${ }^{7}$, les procédés de thématisation ou les reformulations, ainsi que des dimensions paratextuelles comme le thème figurant dans la ligne objet du message et le fait qu'il soit

\footnotetext{
${ }^{7}$ Reprise d'un segment de discours (antécédent) par un mot anaphorique.
} 
précédé ou non par un «re:» qui indique une réplique à un message précédant portant sur le même thème (cf. Mondada, 1999 : 9).

Le discours reporté, donc une citation du message précédent ou de sa partie, insérée ensuite dans le corps de l'e-mail ou d'une contribution de forum, produit un effet de loupe - pour construire son apport discursif, le sujet parlant s'appuie sur les mots déjà dits. Il les évoque, rappelle, observe de près - ceci est bien visible surtout dans les citations des fragments de messages antérieurs :

Réponse de <alain> (A.II.5c) :

bonjour a toi mary je repond a ton mail alors mes fille s ont isabelle23ans virginie 21 ans et priscillia 13 ans dit moi a tu des pation moi je construit des maquette de bateau et a avion et je suis tres cinefil jai une collection de dvd de $500 \mathrm{film}$ alant de edit piaf a defunes fernandel de 1 horreur enfin un peut de tous je te quite en esperant te lire bientot alain $h$

(message incorporé, antérieur par rapport à celle d'au-dessus):

mary anne <maryanne54ly...> wrote :

Alain, merci de m'avoir répondu!! c'est vrai que nous ne sommes pas trés loin!! je suis tout à fait d'accord pour que nous devenions amis; dis moi,guel âge ont tes filles?? je te souhaite un bon dimanche et à bientôt, Maryanne

\section{STRATÉGIES CONVERSATIONNELLES}

Nous voudrions mentionner en quelques mots le problème des stratégies communicatives qui existent quand une interaction se produise au sein d'un groupe d'interlocuteurs intéressés par un sujet donné. Ceci est sans doute l'une des marques de l'oralité des échanges communicatifs dans le cyberespace. La stratégie conversationnelle, d'après Habrajska (2002 : 161), consiste en la production par l'émetteur et par le récepteur d'une suite cohérente des actes de parole, par laquelle les interlocuteurs parviennent à un but communicatif commun. Dans les démarches communicationnels des co-scripteurs, nous verrions souvent une sorte d'anti-stratégie conversationnelle, parce qu'on peut observer plusieurs situations où un interlocuteur bloque les intentions communicatives impliquées par son adversaire, ou bien il mène le fil conversationnel à un but opposé à celui qui a été envisagé par l'adversaire.

Awdiejew (1991) et Habrajska (op. cit. : 168-173) parlent de deux types de réactions d'interlocuteurs aux apports dialogiques : le contact bona fide et le contact non bona fide. Le contact bona fide qui s'effectue quand les sujets interagissant suivent les règles de politesse, de coopération et d'économie communicative, relève 
de deux stratégies : non-antagoniste, quand le locuteur initiant le contact donne l'initiative à son (ses) interlocuteur(s), et antagoniste, quand les interlocuteurs entrent en discussion - échange d'arguments, de vues contradictoires. Le contact non bona fide, quand les règles de coopération ne sont pas suivies, repose soit sur l'échange humoristique des propos en but de s'amuser, soit sur le conflit causé par des émotions négatives dont le bu est une dégradation verbale du partenaire. C'est donc une anti-stratégie conversationnelle.

Une sorte de stratégie négative, une anti-stratégie, fréquente dans les forums de discussion, consiste en la provocation langagière. Après avoir posté un message agressif, contenant des opinions extrêmes ou, simplement, de fautes d'orthographe, le " provocateur » déclenche de vives répliques. Dans notre corpus, il n'y en a pas d'exemples évidents ; la question est plus visible dans le domaine des bavardages en direct, où la tension émotionnelle est beaucoup plus forte. Nous en reviendrons dans le chapitre suivant. Cependant, dans le matériel lexical recueilli, nous pouvons rencontrer plusieurs preuves de contact bona fide de type antagoniste, où les interlocuteurs discutent, se disputent même, mais sans prononcer des invectives. Les paroles, émotionnelles, mais gentilles, de Chiara, sont un symbole d'une telle attitude:

A.V.17. Mi rivolgo a tutte quelle persone che si aggirano su questo forum distribuendo critiche ed offese a destra ed a sinistra, senza nemmeno preoccuparsi di sapere se le informazioni in loro possesso sono autentiche oppure no (..) Se credete di avere ragione $(. .$.$) perchè invece di esprimere le$ vostre opinioni su questo forum non scrivete una lettera al giornale?

Malgré une émotionnalité parfois assez forte, les échanges asynchrones A.III et A.IV témoignent de la volonté de suivre les règles de politesse et de coopération discursive. Les internautes présentent leurs opinions en s'attendant les répliques, même celles qui sont peu favorables (contributions de Andrei et de Embea, A.III).

Le contact non bona fide se manifeste à travers les discussions sur la politique, cependant les contributions que nous présentons dans la partie corpus, avaient été examinées et censurćes (sous l'angle du contenu raciste, etc.) par les modérateurs des forums; il est donc impossible d'y trouver des invectives et des propos réellement agressifs. Nous avons trouvé une contribution provocatrice, car aucunement liée à la thématique des rencontres virtuelles du groupe, restée sans réponse: il s'agit de la proposition de contact (érotique) accompagnée de publicité, [cry] A.II.20.

En fin du compte, l'existence des stratégies conversationnelles dans l'échange asynchrone des messages permet d'y voir une interaction à part entière, même si, nous le répétons, le critère de la co-présence physique n'est pas tenu. Cela confirme le point de vue, selon lequel un échange de lettres électroniques peut être considéré 
comme un dialogue entre deux ou plusieurs participants. L'incorporation de traits conversationnels dans un échange des messages écrits asynchrone, observable dans les stratégies conversationnelles envisagées par des participants, permet de dire encore une fois que l'oralité secondaire se manifeste aussi dans le cyberespace.

\section{EN GUISE DE CONCLUSION}

Dans le présent article, nous avons présenté le phénomène de l'oralité des échanges communicatifs écrits asynchrones, du cyberespace français et italien, à l'exemple des contributions aux forums de discussion. Nous avons posé la lettre électronique e-mail et le message d'un forum de discussion au même rang, celui de la correspondance via Internet, où les réponses sont produites en différé. Il faudrait y joindre également le texto ( $\mathrm{sms}$ ), le court message transmis par le réseau gsm (qui appartient au cyberespace dans son sens large), toutefois nous en parlons succinctement, à titre indicatif, uniquement pour mettre en évidence la spécificité de la forme épistolaire qui, dans le cybermonde, change en forme plurilogale, une interaction à part entière.

La lettre électronique est un message original et complexe, car elle est le résultat des procédés qui mêlent des rituels énonciatifs écrits et oraux. Il ne faut pas avoir une grande pratique pour produire un message conforme à l'esprit du réseau, toutefois c'est dans le contenu de la lettre que l'on observe l'adaptation de l'émetteur aux normes communicationnelles du cyberespace. Une lettre électronique est un ensemble condensé d'exemples de l'emploi des particularités discursives propres à Internet, en commençant par des spécificités graphiques (p.ex. émoticones, signature, forme épistolaire nouvelle), en passant par la morpho-syntaxe (présence de multiples traits oraux comme l'orthographe phonétisante ou le style émotionnel propre à l'énonciation orale), pour aboutir enfin aux particularités énonciatives qui approchent un échange des mails d'un dialogue asynchrone.

Nous avons voulu démontrer qu'un échange des mails est en fait une sorte de dialogue asynchrone, c'est-à-dire ses apports dialogiques sont produits au fur et à mesure de l'accès des sujets interagissant au forum. Les réponses en différé, recueillies dans la partie A.I-V de notre corpus lexical, sont d'un côté considérées comme des formes épistolaires, relevant du nouveau genre appelé messiel (cf. Cusin-Berche, 1999). Elles suivent en général les conventions traditionnelles qui régissent la correspondance, à savoir les exigences formelles du paratexte, même si les formules d'ouverture et de clôture sont réduites, voire absentes, ou le format de date et d'adresse est soumis au système d'envoi des logiciels comme Outlook Express ou celui du web forum. Mais de l'autre côté, l'échange des messages asynchrones crée une situation énonciative semblable à l'interaction en face à face, donc au dialogue oral. Les contributions au forum contiennent souvent une partie du discours de l'autre, énonciateur second: le discours rapporté, auquel l'auteur du 
message, énonciateur premier, répond (surtout A.II). Ainsi, l'internaute introduit une interactivité dans la lettre électronique et construit un espace d'intersubjectivité qui peut se voir modifié par d'autres sujets interagissants, participants de la même discussion dans un forum donné.

Il serait injustifié de voir dans l'échange asynchrone des messages électroniques une nouvelle forme de conversation. La conversation c'est l'interaction pure cette constatation banale ne fait que souligner l'exigence du critère fondamental, celui de la co-présence des sujets interagissant. Ce n'est pas le cas, d'après la définition-même, de l'échange asynchrone. Là, on a affaire à une situation énonciative différente de la conversation quotidienne: la réponse de l'interlocuteur à un message est différée dans le temps. La notion d'échange asynchrone renvoie cependant à celle de dialogue, qui, en restant une sorte d'interaction, n'est pas si strictement limité par l'encadrement temporel.

Pour être encore plus précise, s'il y en a lieu dans cette conclusion partielle, nous devrions expliquer pourquoi nous nous référons au dialogue qui, dans le sens originel du mot, est un entretien entre deux personnes, contact et discussion entre deux parties à la recherche d'un compromis. Il serait plus conforme de parler d'une forme plurilogale, étant donné que, dans l'interaction asynchrone, ce sont le plus souvent plusieurs énonciateurs qui s'envoient les messages. Néanmoins, nous avons précisé, aux débuts du présent chapitre, que le point de départ pour nos réflexions serait une relation entre deux sujets parlant. Quel que soit le circuit de production et de diffusion, la rédaction des messages à délais de réception variables requiert un formalisme épistolaire d'une part, de l'autre le respect des règles de la conversation en face à face. Du point de vue de la recherche de traits oraux le type de l'interaction n'a pas d'importance. Nous prenons donc toutes les formes d'échange asynchrone des messages pour une sorte de dialogue, en s'approchant ainsi des synonymes du mot comme le colloque (débat entre plusieurs personnes), la conversation. et même, si on prend en considération la construction du sens global, la négociation qui est indispensable pour aboutir à la conclusion d'un accord.

La chaine des contributions de l'espace dialogal asynchrone est basée sur le principe de l'implicativité séquentielle. Les relations qui s'établissent dans la temporalité de l'alternance de la parole d'un locuteur à un autre sont en effet constructives pour l'organisation de chaque dialogue, y compris l'interaction asynchrone. L'interlocuteur, dans son intervention, complète le message initial d'un nouveau contenu sémantique mais procède aussi à la création des attentes et contraintes normatives. Il existe donc un certain contrôle de l'espace intersubjectif qui est de facto le contrôle de la compréhension publique.

A notre avis, le fonctionnement du discours dans le cyberespace, au sein des échanges communicatifs asynchrones, fait preuve de l'existence d'une oralité secondaire, parallèle à la langue écrite et résultante de celle-ci. Puisque nous 
associons la notion d'oralité à l'écrit aux faits du paralangage ${ }^{8}$, nous pouvons dire que le courrier électronique appartient aux types de discours d'une forte tension affective, parsemés de traits d'oralité propres au langage parlé. Dans notre corpus, il y a des exemples de messages de styles différents - de la correspondance privée dont les phrases sont caractéristiques à la syntaxe des énoncés oraux (A.II), jusqu'à l'échange d'idées sous forme élaborée, propre à une discussion ordonnée, méthodique (A.III - V). Dans l'échange des mails des stratégies de politesse, à l'image de celles du genre épistolaire traditionnel, sont employées et respectées, bien qu'elles se voient modifiées par le médium électronique - le style et la forme de la communication médiée par ordinateur étant pour toujours moins exigeants et moins rigides.

Les forums de discussion font certainement preuve de l'existence d'un humour in-group language, toutefois notre corpus ne nous a pas apporté d'exemples adéquats. Nous pouvons parler d'une certaine ironie et amertume des énonciateurs à propos de la situation politique à laquelle ils se référent dans leurs contributions (surtout A.III - V), mais il est impossible d'y observer un jeu de mots et des amusements lexicaux propres aux usages établis par une communauté de gens qui se connaissent ou qui s'expriment sur des sujets comiques.

Tout compte fait, nous soutenons que l'échange asynchrone des messages-lettres électroniques permet de recenser plusieurs manifestations de l'oralité qualifiée par nous comme secondaire, car dépendante de l'écriture. Le phénomène de l'oralité secondaire est parfaitement visible dans le discours du cyberespace, dans la structure morphosyntaxique tout comme dans l'organisation énonciative séquentielle des échanges communicatifs asynchrones.

\section{BIBLIOGRAPHIE}

Anis J. (1999), Chats et usages graphiques du français, dans : J. Anis, Internet, communication et langue française, Hermes Sciences Publications, Paris.

Awdicjew A. (1991), Strategie konwersacyjne (próba typologii), Socjolingwistyka, XI, pp. 7־20.

Cus in-B erche F. (1997), A la recherche de quelques caractéristiques linguistiques des textes spécialisés et de la rédaction technique, Langage et l'homme, vol. XXXII, $\mathrm{n}^{\circ} 4$, De Boeck, Bruxelles 1997, pp. 21.41.

Cusin-Berche F. (1999), Courriel et genres discursifs, dans : J. Anis, Internet, communication et langue française, Hermes Sciences Publications, Paris.

Grice P.H. (1979), Logique et conversation, Communications, $n^{0} 30$, Seuil.

Habrajsk a G. (2002), Strategie konwersacyjne w Internetowych grupach dyskusyjnych, „Język a komunikacja 4" : Język trzeciego tysiqclecia, (opublikowane materialy z konferencji Kraków 28 lutego - 2 marca 2002), Tertium, Kraków, p. 161-174.

${ }^{8}$ Le paralangage, c'est le moyen de communication naturel non langagier, employé seul ou plus généralement simultanément avec la parole. A l'oral, ce sont : la mimique, la gestuelle, l'intonation, les pauses; à l'écrit : la mise en page, la typographie porteuse de sens, la ponctuation au sens large, les images et les couleurs. 
$\mathrm{H} \mathrm{crt}$ Ph. (1997), Quasi-oralité de l'écriture électronique et sentiment de communauté dans les débats scientifiques en ligne, Réseaux, $\mathrm{n}^{\circ}$ 97, http: //www.enssib.fr/autres-sites/reseaux-cnet/, (vérification du site : avril 2005).

Mondada L. (1999), Formes de séquentialité dans les courriels et les forums de discussion, http: //alsic.u-strasbg.fr, vol. 2, numéro 1 (vérification du site : avril 2005).

Mourlhon-Dallies F., Colin J.-Y. (1995), Les rituels énonciatifs des réseaux informatiques entre scientifiques, Les carnets du Cediscor, $\mathrm{n}^{\circ}$ 3, Presses de la Sorbonne Nouvelle, Paris, pp. 161-172.

Orlikowski W., Yates J. (1994), Genre repertoire: The structuring of communicative practices in organizations, Administrative Science Quarterly, 39(4) : 541-574.

P i rogowsk a E. (2005), L'oralité secondaire dans le discours du cyberespace français et italien, thèse de doctorat soutenue à l'Université Adam Mickiewicz, dir. J. Sypnicki, Poznań.

Schegl off E., Sacks H. (1973), Opening up closing, Semiotica, 8, p. 289-327. 